\title{
MBE GROWTH AND PROPERTIES OF ZnYbTe LAYERS
}

\author{
J. Sadowski, K. Szamota-Sadowska, K. Świątek, L. KowalczyK \\ Institute of Physics, Polish Academy of Sciences \\ Al. Lotników 32/46, 02-668 Warszawa, Poland
}

A. Abounadi and A. Rajira

Université Montpellier 2, Place E. Bataillon, Montpellier, France

The MBE grown ZnYbTe layers were characterized by X-ray diffraction, photoluminescence and reflectivity measurements. The MBE growth conditions allowing to obtain monocrystalline $\mathrm{ZnYbTe}$ layers were found to be metal-rich (MBE growth with excess of $\mathrm{Zn}$ flux). In optical measurements (photoluminescence, reflectivity), both transitions connected with ternary $\mathrm{ZnYbTe}$ compound and with $\mathrm{Yb}^{3+}$ ions were detected. The quality of $\mathrm{ZnYbTe}$ layers with $\mathrm{Yb}$ content of $3 \%$ and $1 \%$ is inferior to the quality of pure $\mathrm{ZnTe}$ MBE layers, which is clearly seen in the results of photoluminescence and reflectivity measurements. In the $\mathrm{ZnYbTe}$ layers with $3 \%$ $\mathrm{Yb}$, exhibiting monocrystalline character in reflection high-energy electron diffraction and X-ray diffraction measurements, optical transitions characteristic of pure $\mathrm{YbTe}$ were detected. In $\mathrm{ZnYbTe}$ layers with $1 \% \mathrm{Yb}$, no transitions connected with YbTe were observed.

PACS numbers: 81.15.Gh, 61.14.Hg

\section{Introduction}

Because of different crystallographic structure of II-VI (zinc blende) and rare earth (RE) tellurides (rock salt) it is difficult to obtain ternary II-RE-Te alloys. Bulk crystal growth methods allow one to obtain II-VI compounds doped with rare earth elements with Re concentrations of $10^{17}-10^{20} \mathrm{~cm}^{-3}$ [1]. Using MBE crystallization technique it is possible to obtain monocrystalline layers of RE alloyed II-VI compounds with RE concentrations of a few percent [2]. We present here the properties of MBE grown $\mathrm{ZnTe}$ layers alloyed with $\mathrm{Yb}$, with $\mathrm{Yb}$ content of $1 \%$ to $3 \%$. The solubility limit of $\mathrm{Yb}$ in $\mathrm{ZnTe}$ was evaluated previously [2] to be about $3 \%$. 


\section{MBE growth of ZnYbTe layers}

MBE growth processes were performed in a two chamber MBE growth system manufactured in the Institute of Physics of the Polish Academy of Sciences in Warsaw. As substrates the Freiberger epi-ready, semi-insulating $\mathrm{GaAs}(100)$ wafers, $2^{\circ}$ off-oriented towards [011] direction were used. After the thermal oxide desorption by heating to $585^{\circ} \mathrm{C}$ the substrates were cooled to the $\mathrm{ZnTe}$ buffer growth temperature $\left(320^{\circ} \mathrm{C}\right)$ in $\mathrm{Zn}$ flux. The MBE growth of $\mathrm{ZnTe}$ buffers and $\mathrm{ZnYbTe}$ layers was performed in Te-rich (sample 1), or Zn-rich conditions (samples 2, 3 and 4). The parameters of samples are listed in Table. The excess of $\mathrm{Zn}$ or Te flux

TABLE

Parameters of $\mathrm{ZnYbTe}$ layers crystallized on the GaAs(100) substrates.

\begin{tabular}{c|c|c|c|c|c}
\hline \hline Sample No. & $\begin{array}{c}\text { ZnTe buffer } \\
\text { thickness }\end{array}$ & $\begin{array}{c}\text { ZnYbTe } \\
\text { thickness }\end{array}$ & $\begin{array}{c}\text { ZnTe cap layer } \\
\text { thickness }\end{array}$ & Yb content & $\begin{array}{c}\text { Growth } \\
\text { conditions }\end{array}$ \\
\hline 1 & $1.5 \mu \mathrm{m}$ & $1 \mu \mathrm{m}$ & 0 & $3 \%$ & Te-rich \\
2 & $3.5 \mu \mathrm{m}$ & $100 \AA$ & $1800 \AA$ & $1 \%$ & Zn-rich \\
3 & $1.5 \mu \mathrm{m}$ & $100 \AA$ & $1200 \AA$ & $1 \%$ & Zn-rich \\
4 & $1.5 \mu \mathrm{m}$ & $1200 \AA$ & 0 & $3 \%$ & Zn-rich
\end{tabular}

was controlled by choosing the value of $\mathrm{Te} / \mathrm{Zn}$ flux ratio for which the $(2 \times 1) \mathrm{Te}$ or $c(2 \times 2) \mathrm{Zn}$ reconstruction of the $\mathrm{ZnTe}$ buffer layer surface was observed. As the MBE growth temperature of $\mathrm{ZnTe}$ and $\mathrm{ZnYbTe}$, the optimal temperature for growth of $\mathrm{ZnTe} \mathrm{MBE}$ layers was chosen (about $320^{\circ} \mathrm{C}$ ). For all the samples the streaky reflection high-energy electron diffraction (RHEED) patterns were observed after growing the first $600 \AA$ of $\mathrm{ZnTe}$ buffer. During the $\mathrm{ZnYbTe}$ growth in Te-rich conditions, the streaky RHEED patterns turned to the spotty ones after growing of about $100 \AA$ thick $\mathrm{ZnYbTe}$ and when the film thickness increased, the diffraction picture typical of textured layers was observed. In the case of samples 2,3 , and 4 , the streaky RHEED patterns were observed during the whole growth process.

\section{X-ray diffraction measurements}

All samples were measured by X-ray diffraction system with $\mathrm{Cu} K_{\alpha_{1}} K_{\alpha_{2}}$ incident beam. Sample 1 was measured in the polycrystalline scan mode, samples 2, 3, 4 in rocking curve configuration. Sample 3 was also measured by the 4-crystalline diffractometer. For sample 1 the broad diffraction peak connected with $\mathrm{ZnYbTe}$ was observed, with additional peak resulting from pure YbTe rock-salt phase. In the case of samples 2, 3 and 4 only diffraction peaks from $\mathrm{ZnTe}$ buffer layers were seen (due to the small thickness and little difference between $\mathrm{ZnTe}$ and $\mathrm{ZnYbTe} \mathrm{lattice} \mathrm{constants).} \mathrm{The} \mathrm{full} \mathrm{width} \mathrm{at} \mathrm{half} \mathrm{maximum} \mathrm{of} 400 \mathrm{Bragg}$ reflection from $1.5 \mu \mathrm{m}$ thick $\mathrm{ZnTe}$ buffer of sample 3 was about 200 arc s, which indicates a good quality of $\mathrm{ZnTe} / \mathrm{GaAs}(100)$ buffer. 


\section{Optical measurements}

The photoluminescence (PL) experiments were performed using a double-grating GDM-1000 monochromator and EMI 9558 S20 photomultiplier. Investigated samples were mounted in a Leybold closed-cycle cryostat and illuminated by 458,488 or $514 \mathrm{~nm}$ lines of an Ar laser. Figure 1 shows a comparison of PL spectra

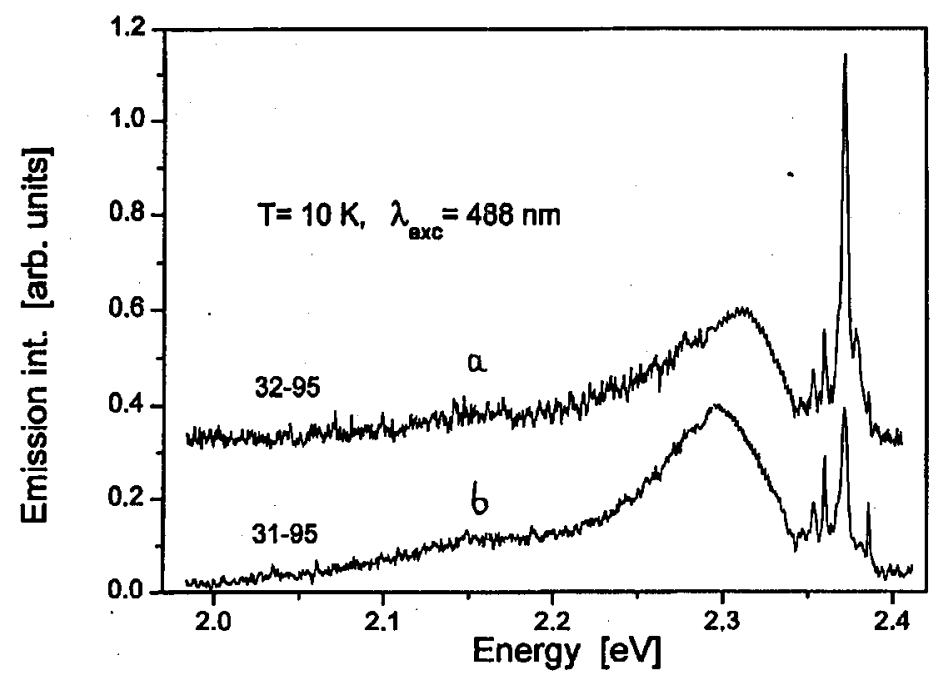

Fig. 1. Low temperature PL spectrum of $\mathrm{ZnYbTe}$ layers: (a) $1200 \AA$ thick $\mathrm{Zn}_{0.97} \mathrm{Yb}_{0.03} \mathrm{Te}$ - sample 4, (b) $100 \AA$ thick $\mathrm{Zn}_{0.99} \mathrm{Yb}_{0.01} \mathrm{Te}$ - sample 3.

of two ZnYbTe layers: (a) sample 3, (b) sample 4, with different thicknesses and $\mathrm{Yb}$ concentration, measured at $10 \mathrm{~K}$ under $488 \mathrm{~nm}$ laser light excitation. A set of narrow emission peaks in the energy range $2.35-2.4 \mathrm{eV}$ was detected. The near band-gap luminescence of $\mathrm{ZnYbTe} / \mathrm{ZnTe}$ (sample 3) structures exhibits a weak free exciton PL split into a heavy- and light-hole exciton at 2.385 and $2.378 \mathrm{eV}$, respectively. This splitting is probably caused by strain. We assign a set of three sharp PL peaks in the energy range $2.35-2.375 \mathrm{eV}$ to recombination of excitons bound to neutral acceptors. A strong luminescence at approximately $2.372 \mathrm{eV}$ is most probably related to recombination of nitrogen bound exciton. The strong and wide emission with maximum at $\approx 2.3 \mathrm{eV}$, seen in both samples (Fig. 1a, 1b), comes from the $\mathrm{ZnYbTe}$ layer. Its energy is situated between band-gap energy of pure $\mathrm{ZnTe}(2.396 \mathrm{eV})$ and $\mathrm{YbTe}(1.97 \mathrm{eV})$ crystals. Large inhomogeneous broadening of this emission indicates a significant fluctuations of $\mathrm{Yb}$ concentration present in the investigated ZniYbTe layers. Moreover, in infrared region of PL spectra of some samples we have found a weak emission at $\approx 1.27 \mathrm{eV}$ related most probably to the $\mathrm{Yb}^{3+} 4 f^{13}\left({ }^{2} F_{5 / 2}{ }^{2} F_{7 / 2}\right)$ intra-ion transition.

At high excitation power density (using 3rd harmonic of Nd:YAG laser) a remarkable shift of the $\mathrm{PL}$ signal connected with $\mathrm{ZnYbTe}$ (wide maximum about $2.3 \mathrm{eV}$ ), towards higher energies was observed. 


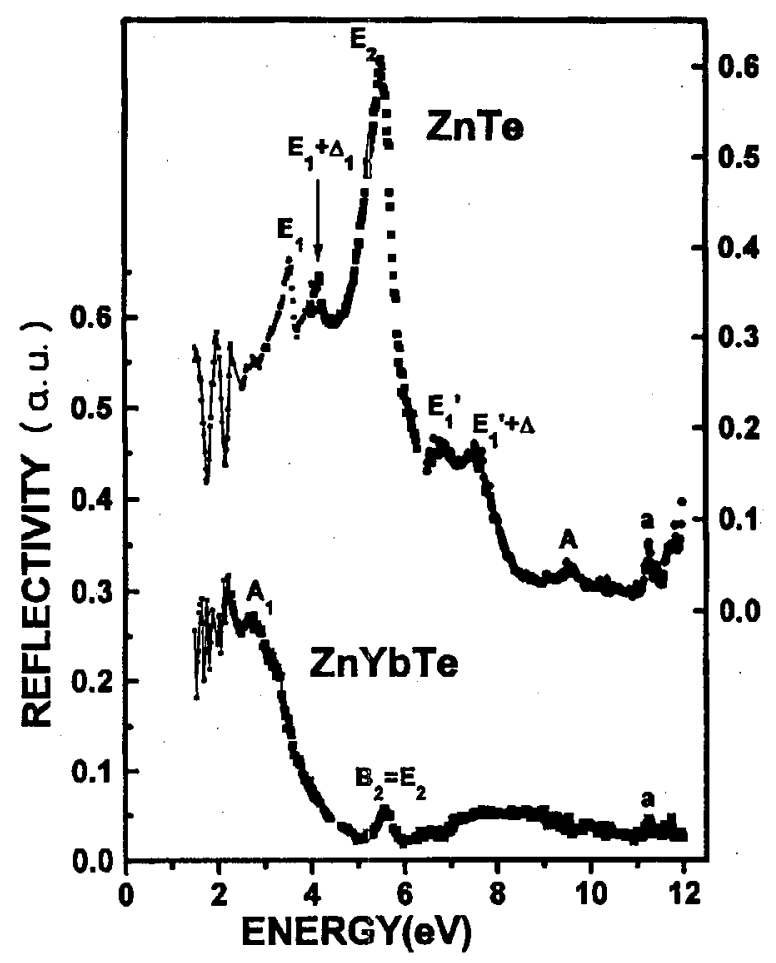

Fig. 2. Room temperature reflectivity of $\mathrm{MBE}$ grown $\mathrm{ZnTe}$ and $1200 \AA$ thick $\mathrm{Zn}_{0.97} \mathrm{Yb}_{0.03} \mathrm{Te}$ layer - sample 4 .

Figure 2 presents the room temperature (RT) reflectivity spectra obtained for energies in the range 1.5-12 eV for pure $\mathrm{ZnTe}$ and $\mathrm{ZnYbTe}$ (sample 4) layer. The details of the detection system are given elsewhere [2]. The results are presented in the sequence which allows one to compare spectra obtained for $\mathrm{ZnTe}$ and $\mathrm{ZnYbTe}$. The reflectivity peaks were labeled as: $E_{1}$ at $3.53 \mathrm{eV}, E_{1}+\Delta_{1}$ at $4.101 \mathrm{eV}, E_{2}$ at $5.5 \mathrm{eV}, E_{1}^{\prime}$ at $6.8 \mathrm{eV}, E^{\prime}+\Delta$ at $7.52 \pm 0.2 \mathrm{eV}, A$ at $9.5 \mathrm{eV}$ and $a$ at $11.26 \mathrm{eV}$. Maxima $E_{1}$ and $E_{1}+\Delta_{1}$ were assigned to transition in $\Lambda$ direction in Brillouin zone [3]. The $E_{2}$ maximum was attributed [3] to the transition at the $X$ point in Brillouin zone and $E^{\prime}$ as well as $E_{1}^{\prime}+\Delta$ were attributed [3] to the transitions at the $L$ point. In the case of $\mathrm{ZnYbTe}$ layer (sample 4), the transition at the $X$ point is marked by $A_{1}$ [4]. The experimental values of these maxima are in accordance with the published ones. A significant change in behavior of the $\mathrm{ZnYbTe}$ spectrum in comparison to the pure $\mathrm{ZnTe}$ can be seen. For $\mathrm{ZnYbTe}$, the shoulder appears between 3 and $4 \mathrm{eV}$. It can be caused by two effects: the transition observed for pure $\mathrm{YbTe}$ in this energy region [5], or the transition visible for $\mathrm{ZnTe}$ at $3.53 \mathrm{eV}$. The peak marked as $A$ can be assigned to the transition characteristic of pure YbTe [4]. It seems that the peak named $B$ is connected with the same transition as the one named $E_{2}$ for $\mathrm{ZnTe}$. The $a$ peak detected for $\mathrm{ZnYbTe}$ is also similar to the same peak observed in the case of $\mathrm{ZnTe}$. 
The important effect observed for measured $\mathrm{ZnYbTe}$ samples was the decrease in the reflectivity signal. As we know, in the UV energy region decay of the oscillator strength of the valence to conduction band transition [4] takes place, on the other hand, RE atoms usually occupy more than one type of the site in zinc blende crystal lattice [5]. Therefore the decrease in reflectivity is probably caused by these two effects.

\section{Discussion}

In the case of $\mathrm{ZnYbTe}$ films with $3 \% \mathrm{Yb}$, optical transitions characteristic of pure YbTe rock-salt phase were detected for both films grown in Zn-rich and in Te-rich conditions. However, during MBE growth process the streaky RHEED patterns, indicating smooth monocrystalline $\mathrm{ZnYbTe}$ surface, were observed only for layers grown in the $\mathrm{Zn}$-rich conditions. In the case of sample 3, the heavy hole (hh)-light hole (lh) exciton recombination energy splitting of the ZnYbTe cap layer was observed, which indicates that this layer is partially strained. As the thickness of $\mathrm{ZnYbTe}$ layer is only $100 \AA$ in this case and the lattice constant difference between $\mathrm{ZnTe}$ and $\mathrm{ZnYbTe}$ with $1 \% \mathrm{Yb}$ is very little (about $0.002 \AA$ ), the strain is already present in $1.5 \mu \mathrm{m}$ thick $\mathrm{ZnTe}$ buffer (it is known from literature: e.g. [6] that even about $3.5 \mu \mathrm{m}$ thick $\mathrm{ZnTe}$ layers crystallised on GaAs substrates are still not strain-free). The well-resolved lh-hh exciton lines of the $1800 \AA$ thick $\mathrm{ZnTe}$ cap layer prove the high quality of the whole structure ( $\mathrm{ZnTe}$ buffer $/ 100 \AA$ thick $\mathrm{ZnYbTe}$ layer $/ 1800 \AA$ thick ZnTe cap).

\section{Conclusions}

The monocrystalline $\mathrm{ZnYbTe}$ films with $\mathrm{Yb}$ concentrations up to $3 \%$ can be grown by $\mathrm{MBE}$ in $\mathrm{Zn}$-rich conditions. In the films with $3 \% \mathrm{Yb}$ content the optical transitions characteristic of pure YbTe were observed. In the $100 \AA$ thick $\mathrm{ZnYbTe}$ layers with $1 \% \mathrm{Yb}$ no traces of $\mathrm{YbTe}$ inclusions were found, however the quality of the layers estimated by optical methods is inferior to the quality of pure ZnTe layers. XRD measurements and RHEED diffraction pictures showed a much better crystalline quality of ZnYbTe layers grown in Zn-rich conditions in comparison to the layers grown in Te-rich conditions, however in RT reflectivity and low temperature PL measurements no big differences between these layers were observed. In all the layers weak $\mathrm{PL}$ signal connected with $\mathrm{Yb}^{3+}$ ion transitions was detected.

\section{References}

[1] R. Boyn, Phys. Status Solidi B 148, 11 (1988).

[2] J. Sadowski, E. Dynowska, K. Szamota-Sadowska, W. Przedpełski, P. Sitarek, K. Świątek, J. Cryst. Growth 159, 1075 (1996).

[3] M. Cardona, D.L. Greenaway, Phys. Rev. 131, 98 (1963).

[4] A. Kisiel, M. Zimnal-Starnawska, F. Antonangeli, M. Piacentini, N. Zema, Il Nuovo Cimento D 8, 436 (1986).

[5] R. Suryanarayanan, J. Ferre, B. Briat, Phys. Rev. B 9, 554 (1974).

[6] M. Averous, A. Abounadi, R.L Aulombard, D. Bouchara, N. Briot, O. Briot, J. Calas, B. Gil, Phys. Status Solidi B 181, 427 (1994). 